\title{
Enhanced Acoustic Cavitation Following Laser-Induced Bubble Formation: Long-Term Memory Effect
}

\author{
Oguz Yavas and Paul Leiderer \\ Department of Physics, University of Konstanz, D-78434 Konstanz, Germany \\ Hee K. Park and Costas P. Grigoropoulos \\ Department of Mechanical Engineering, University of California, Berkeley, California 94720 \\ Chie C. Poon and Andrew C. Tam \\ IBM Almaden Research Center, 650 Harry Road, San Jose, California 95120-6099
}

(Received 27 September 1993)

\begin{abstract}
The enhancement of acoustic cavitation at a liquid-solid interface following laser-induced bubble formation is studied. The experimental results indicate that metastable ultramicroscopic bubbles formed on the solid surface cause a long-term "memory effect" on acoustic cavitation. By performing a doublepulse experiment using two excimer lasers, the temporal decay of this memory effect is determined for two different liquids on a chromium surface. An explanation of the observed decay mode by a diffusion model is presented.
\end{abstract}

PACS numbers: 47.55.Bx, 62.60.+v, 64.60.- $\mathrm{i}$, 64.70.Fx

Acoustic cavitation, i.e., the formation of bubbles or cavities in a liquid induced by the tensile pressure of an acoustic field, is of interest for a better understanding of the liquid-vapor phase-change phenomenon. It is also of importance in many technical applications, such as sonochemistry, ultrasonic cleaning, laser surgery in medicine, and lithotripsy [1-4]. The inception of cavitation at acoustic amplitudes far below the theoretically predicted tensile pressure has been explained by the hypothesis that long-lived cavitation nuclei, such as ultramicroscopic bubbles or other inhomogeneities, are usually present in liquids [5]. However, small bubbles are thermodynamically unstable and may either dissolve and vanish quickly or grow and rise to the liquid surface [6]. Therefore, several models have been suggested to account for the generation and stabilization of microbubbles, including cosmic radiation $[5,7,8]$, clusters of organic or ionic molecules [9-11], van der Waals stabilization [12], and gas inclusion in crevices on container wall or solid impurities in the liquid [13,14]. In addition, for highly purified and degassed water a significant decrease of the cavitation threshold due to neutron irradiation has been observed. This decrease is reversible; i.e., the threshold returns to the initial level in about half an hour when the source of radiation is removed [5], which is an example of a memory effect.

The physical mechanism responsible for this memory effect has not been understood, and, to our knowledge, there has not been any study on a possible memory effect for acoustic cavitation at a liquid-solid interface. The time scale for previous studies has been limited to microseconds due to the instability of the transducers to generate shorter acoustic pulses of sufficient intensities to cause cavitation. Here, we demonstrate the generation of acoustic cavitation by nanosecond pulses and show for the first time the existence of a bubble memory effect at a liquid-solid interface. The temporal decay of this memory effect has been measured and can be explained by a diffusion process.

Recently, we have demonstrated that the optical reflectance probe is a powerful tool for the nanosecond time scale studies of laser-induced nucleation and growth of bubbles at a liquid-solid interface [15]. The same approach is applied to the acoustic cavitation studies in the present work. The optical arrangement is indicated in the inset in Fig. 1. Further details of the experimental setup appear elsewhere [16]. A thin chromium film has been used as an absorbing solid sample because it has no intrinsic thermoreflectance effects in the fluence range used $\left(\$ 150 \mathrm{~mJ} / \mathrm{cm}^{2}\right)$. Water and methanol are studied as test liquids. The excimer laser irradiation causes the temperature at the solid surface to rise above the boiling temperature of the liquid in about 16 ns. This results in the formation of bubbles at the liquid-solid interface uniformly distributed in the irradiated area, which is monitored by the transient changes in the optical reflectance signal [15]. The excimer laser irradiation also leads to the generation of an acoustic pulse via the thermoelastic effect [17], the intensity of which is strongly increased due to the collective collapse of bubbles. This highintensity acoustic pulse propagates in the liquid, is reflected back to the sample by the quartz window, and causes cavitation at the liquid-solid interface after its round trip in the cuvette. As a result, repetitive "echoes" in the optical reflectance signal are observed when the surfaces of the solid sample and the quartz window are parallel [18]. An example for a methanol-chromium interface is shown in Fig. 1. While the initial reflectance drop is due to bubble formation induced by the excimer laser heating, the succeeding echoes are solely due to 


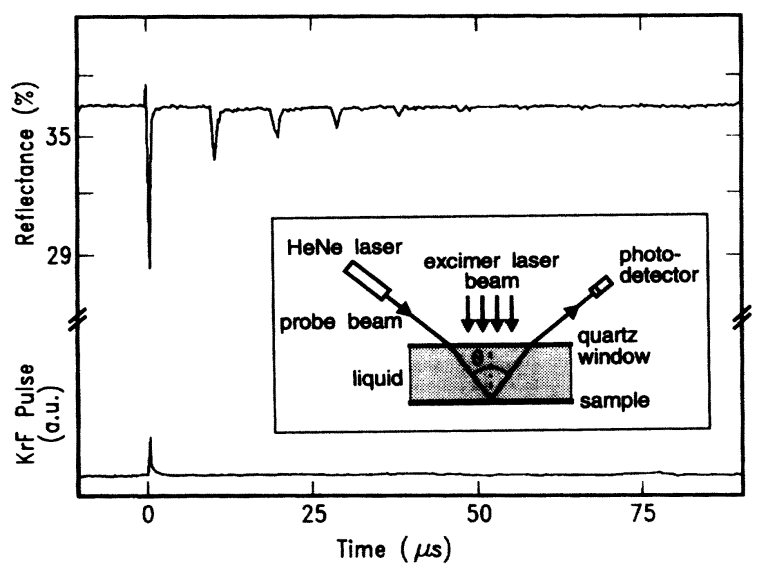

FIG. 1. Optical reflectance signal due to bubble formation at a methanol-chromium interface. $\lambda_{\text {probe }}=632.8 \mathrm{~nm}$, unpolarized, $\theta=15^{\circ}$. The solid sample and the quartz window are parallel as shown in the inset. (The distance between the solid sample and the quartz window is $\sim 7 \mathrm{~mm}$.)

acoustic cavitation induced by the traveling pressure pulse.

Previously, the decrease of the amplitude of these successive echo signals had been ascribed to the attenuation of the acoustic pulse. However, as we shall see, this decrease is also due to the slow decay of the cavitation efficiency, i.e., the memory effect. The memory effect is demonstrated using the experimental configuration shown in Fig. 2(a). The quartz window is tilted with respect to the solid sample so that after a round-trip travel in the cuvette the acoustic pulse is displaced and partly overlaps with the laser-irradiated area. Consequently, there are three distinct regions on the solid surface. Region $I$ is irradiated by the excimer laser pulse only. Region II is irradiated by the excimer laser and additionally subjected to the acoustic pulse with a delay time corresponding to its round-trip period in the cuvette. Region III is free from laser irradiation and is subjected to the acoustic pulse only. The HeNe laser beam is positioned to probe each of the three different regions. Representative results for a water-chromium interface are shown in Fig. 3(a). For region I, only one reflectance signal is observed, which is due to the initial bubble formation induced by the excimer laser heating. For region II, two separate reflectance signals are observed. The first is due to the initial bubble formation and the second is due to acoustic cavitation. A temporal shift of the second reflectance signal with respect to the first is observed as the probe beam is displaced across the sample. It agrees well with the traversed path length of the acoustic wave and the speed of sound in the liquid. For region III, no reflectance signal is observed; i.e., the acoustic pulse cannot cause any cavitation at a previously unirradiated surface. Thus, the laser-induced bubble formation at the solid surface results in a long-term enhancement of acoustic cavitation. Our heat diffusion computations have affirmed that the

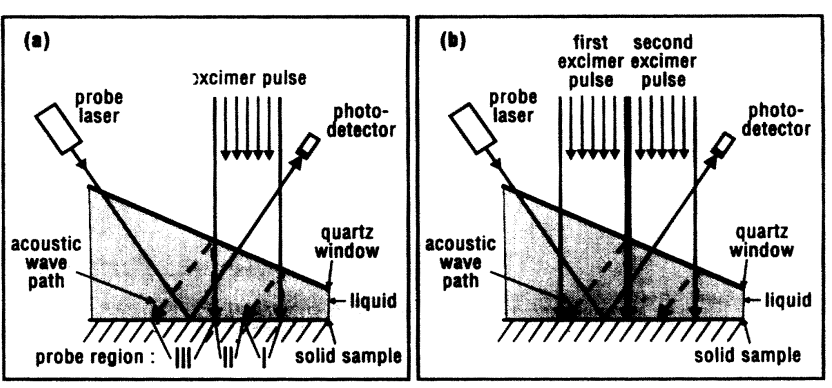

FIG. 2. (a) Experimental setup for proof of the existence of the memory effect. Solid line represents the path of the laser beam, dashed line the path of the acoustic pulse in the liquid. (b) Experimental setup for the measurement of the temporal decay of the memory effect. The first excimer laser initiates the memory effect and the second generates the acoustic pulse.

temperature at the water-chromium interface decreases to room temperature in about $1 \mu$ s after the laser pulse [16]. Consequently, any thermal effect can be excluded as a possible explanation for this memory effect. Therefore, it is hypothesized that the laser-induced bubbles collapse into metastable ultramicroscopic bubbles, i.e., long-lived cavitation nuclei. These nuclei, which are deemed absent on the unirradiated surface, subsequently enhance acoustic cavitation.

In order to determine the temporal decay of the memory effect, we have performed a series of double laser-pulse experiments. The setup of this experiment is shown in Fig. 2(b). The beams from two separate 248$\mathrm{nm} \mathrm{KrF}$ excimer lasers are directed to the solid sample without any spatial overlap. The HeNe laser beam probes the location which corresponds to region I for the first pulse and to region III for the second pulse. The first laser pulse initiates the memory effect and the second
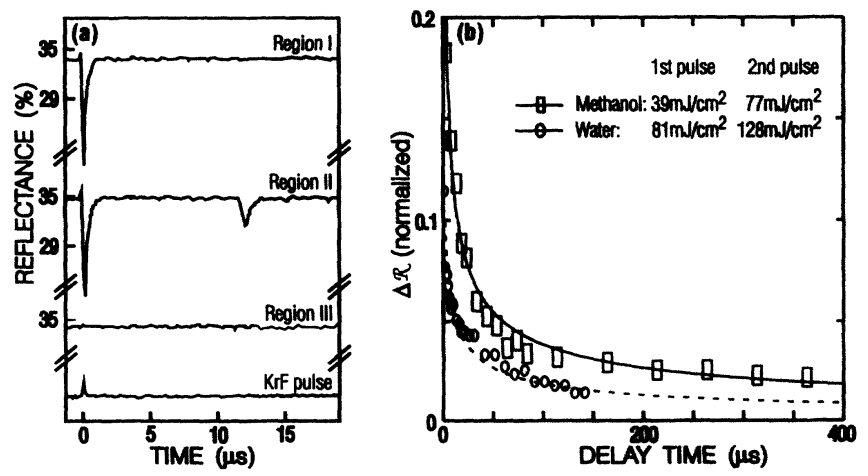

FIG. 3. (a) The optical reflectance signal for the three regions shown in Fig. 2(a) confirms the existence of a memory effect at the laser-irradiated area. (b) The echo signal peak reflectance drop as a function of delay time between the two excimer laser pulses for the water-chromium and methanolchromium interface. The lines represent the fit of the experimental data with the diffusion model. 
pulse generates the high-intensity acoustic pulse. The time delay between the first and the second laser pulses is varied. The fluences are kept constant to assure that both the amount of microbubbles formed and the intensity of the acoustic pulse are about the same. When the two lasers are fired simultaneously, the same signal as in Fig. 3(a) is observed (signal for region II). With increasing delay time the amplitude of the second reflectance signal decreases; i.e., the memory effect decays. Eventually, acoustic cavitation returns to normal efficiency. Representative results for water-chromium and methanolchromium interfaces are shown in Fig. 3(b). The temporal decay in the reflectance signal can be well fitted with the following formula:

$$
\Delta R(t)=C / \sqrt{t+t_{0}},
$$

where $\Delta \mathcal{R}(t)$ is the peak reflectance drop normalized with respect to the initial reflectance value, $C$ the proportionality constant, $t$ the delay time between the laser pulse and the acoustic pulse $(t \geq 0)$, and $t_{0}$ a constant to account for the time required for the laser-induced stabilized nuclei formation. Typical values for $C$ range from 0.1 to $0.2(\mu \mathrm{s})^{1 / 2}$ for water and 0.2 to $0.4(\mu \mathrm{s})^{1 / 2}$ for methanol. The values for $t_{0}$ range from 0.3 to $3.2 \mu \mathrm{s}$ for water and 0.9 to $9.7 \mu \mathrm{s}$ for methanol. These constants are dependent upon the first and the second laser fluences. The fitting equation resembles the well-known equation for the surface history for a one-dimensional diffusion process [19]. Graphing the data in semilogarithmic scales also reveals the $t^{-1 / 2}$ dependence. The decrease in the reflectance signal due to scattering by small nonabsorbing particles in a host medium, such as bubbles in a liquid, is given by [20]

$$
\begin{aligned}
\Delta R(t) & =1-\exp [-\gamma(t) L] \\
& =1-\left[1-\gamma(t) L+\frac{[\gamma(t) L]^{2}}{2 !}-\cdots\right],
\end{aligned}
$$

where $\gamma(t)=\sum_{r} N(t, r) C_{\text {ext }}(t, r)$ is the total extinction coefficient from the collection of bubbles per unit volume of the liquid, $N(t, r)$ is the bubble number density of radius $r, C_{\text {ext }}(t, r)$ is the extinction cross section, and $L$ is twice the thickness of the layer of bubbles in the liquid traversed by the optical beam. Previous works $[6,11]$ have shown that the diffusion velocity for bubbles is small and since the time scale considered is less than hundreds of microseconds, the expected displacement of the bubbles by diffusion is small. Therefore, $L$ is essentially constant.

We note that $C_{\mathrm{ext}} \cong 2 \pi r^{2}$ is the asymptotic limit for Mie scattering which is valid for grown-up bubbles at peak reflectance drop. Also our data show that $\Delta \mathscr{R}(t)$ is generally of the order 0.1 or smaller. With these simplifications, the above equation can be approximated by

$$
\Delta \mathcal{R}(t) \propto \sum_{r} N(t, r) r^{2}(t) .
$$
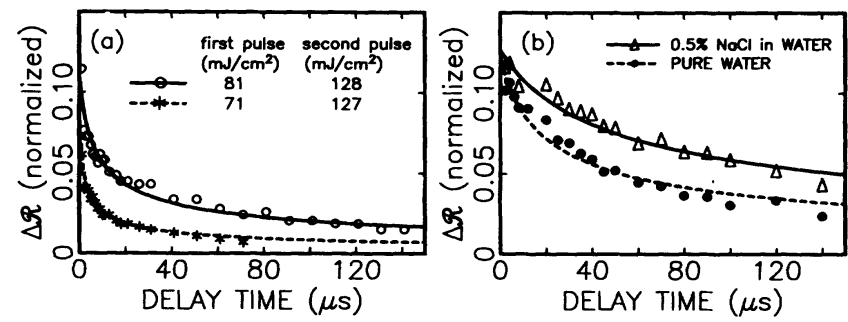

FIG. 4. (a) Effect of fluence variation for the first laser pulse on the memory effect. (b) Stabilizing effect of ion concentration on the temporal decay of the memory effect for waterchromium interface. $F_{1}=82 \mathrm{~mJ} / \mathrm{cm}^{2}$ (first pulse) and $F_{2}=120$ $\mathrm{mJ} / \mathrm{cm}^{2}$ (second pulse). The lines represent the fit of the experimental data with the diffusion model.

The diffusive nature in $\Delta R(t)$ can be interpreted to be caused by three mechanisms. First, the bubble radius can decrease by diffusion of entrapped gases into the liquid. Second, stable microbubbles may diffuse away from the sample interface to yield a smaller bubble number density. However, this effect is expected to be small as described above. The third mechanism is the coalescence of microbubbles to form bigger bubbles. In this respect, the decrease in $N$, dominant over the increase in $r$, produces a net reduction in the extinction cross section. It is not possible from the present data to rank the first and third mechanisms because the bubble size and number density have not been independently determined.

The interpretation of the temporal decay in the reflectance signal by a diffusion process of microbubble properties can be verified by varying the laser fluences in the double-pulse experiments. When the fluence of the first laser is increased more microbubbles will be formed on the solid surface. Similarly, when the fluence of the second laser is increased an acoustic pulse of higher intensity will be generated. Both mechanisms can lead to an increase of cavitation yield. This has indeed been observed as shown in Fig. 4(a) for a water-chromium interface on which the decay of the memory effect is compared for two different fluence values of the first laser pulse. The amplitude of the reflectance drop increases as the laser fluence is increased. The same trend has been observed when the fluence for the second laser is varied. Similar results have been obtained with methanol as a test liquid. We have also bubbled gaseous nitrogen into the liquid and observed no significant change in the memory effect. This lack of change may be due to the presence of atmospheric gases already dissolved into the liquid sample because the cuvette is not sealed. On the contrary, a significant change on the decay rate is observed when the ion concentration of water is increased with $\mathrm{NaCl}$. Figure 4(b) shows the results for pure (spectroscopic grade) water and for water with $\mathrm{NaCl}(0.5 \%)$. As can be seen, the increase in ion concentration has a stabilizing effect on the microbubbles and consequently the memory effect is prolonged. This result can be ex- 
plained by the hypothesis of the ionic cluster formation $[10,11]$ as a possible stabilization mechanism for ultramicroscopic bubbles.

In conclusion, we have demonstrated the existence of a long-term memory effect, i.e., the enhancement of acoustic cavitation at a liquid-solid interface following laserinduced bubble formation. The temporal decay of the memory effect for two different liquids on a chromium surface has been determined and can be explained by a diffusion process.

This work is done in the facilities of the IBM Almaden Research Center. The authors thank I. K. Pour, D. Krajnovich, F. Lee, and W. P. Leung of IBM Almaden Research Center, J. Boneberg of the University of Konstanz, Germany, and Professor A. Z. Akcasu of the University of Michigan, Ann Arbor, for valuable contributions to this work. Support by the Computer Mechanics Laboratory of the University of California at Berkeley is gratefully acknowledged. O. Yavas gratefully acknowledges the fellowship from the Friedrich-Ebert-Stiftung, Bonn, Germany.

[1] F. R. Young, Cavitation (McGraw-Hill, London, 1989), and references therein.

[2] R. E. Apfel, in Methods of Experimental Physics, edited by P. T. Edmonds (Academic, New York, 1981), Vol. 19, p. 355 .
[3] T. J. Mason, Practical Sonochemistry (Ellis Horwood, New York, 1991)

[4] J. Bardina, in Particles on Surfaces, edited by K. L. Mittal (Plenum, New York, 1988), Vol. 1, p. 327.

[5] R. D. Finch, J. Acoust. Soc. Am. 36, 2287 (1964).

[6] P. S. Epstein and M. S. Plesset, J. Chem. Phys. 18, 1505 (1950).

[7] D. Lieberman, Phys. Fluids 2, 466 (1959).

[8] D. Sette and F. Wanderlingh, Phys. Rev. 125, 409 (1962).

[9] F. E. Fox and K. F. Herzfeld, J. Acoust. Soc. Am. 26, 984 (1954).

[10] V. A. Akulichev, Sov. Phys. Acoust. 12, 144 (1966).

[11] N. F. Bunkin and F. V. Bunkin, Sov. Phys. JETP 74, 271 (1992).

[12] R. A. Wentzell, Phys. Rev. Lett. 56, 732 (1986).

[13] M. Strasberg, J. Acoust. Soc. Am. 31, 163 (1959).

[14] A. A. Atchley and A. Prosperetti, J. Acoust. Soc. Am. 86, 1065 (1989)

[15] O. Yavas, P. Leiderer, H. K. Park, C. P. Grigoropoulos, C. C. Poon, W. P. Leung, N. Do, and A. C. Tam, Phys. Rev. Lett. 70, 1830 (1993).

[16] O. Yavas, P. Leiderer, H. K. Park, C. P. Grigoropoulos, C. C. Poon, W. P. Leung, N. Do, and A. C. Tam, Appl. Phys. A (to be published).

[17] A. C. Tam, Rev. Mod. Phys. 58, 381 (1986).

[18] A. Polman, W. C. Sinke, M. J. Uttormark, and M. O. Thompson, J. Mater. Res. 4, 843 (1989).

[19] J. Crank, The Mathematics of Diffusion (Clarendon, Oxford, 1975).

[20] H. C. van de Hulst, Light Scattering by Small Particles (Dover, New York, 1981). 


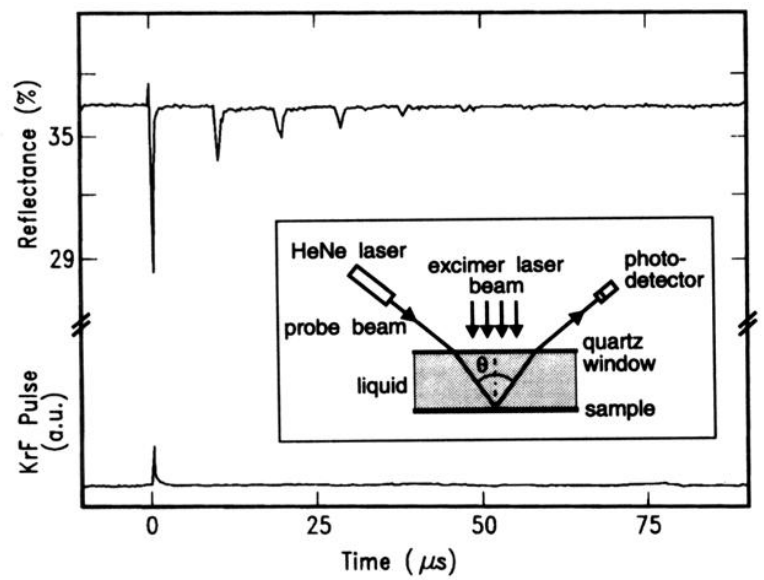

FIG. 1. Optical reflectance signal due to bubble formation at a methanol-chromium interface. $\lambda_{\text {probe }}=632.8 \mathrm{~nm}$, unpolarized, $\theta=15^{\circ}$. The solid sample and the quartz window are parallel as shown in the inset. (The distance between the solid sample and the quartz window is $\sim 7 \mathrm{~mm}$.) 

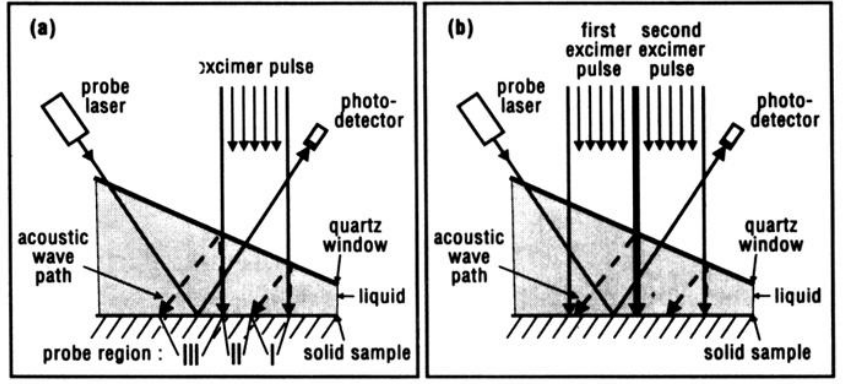

FIG. 2. (a) Experimental setup for proof of the existence of the memory effect. Solid line represents the path of the laser beam, dashed line the path of the acoustic pulse in the liquid. (b) Experimental setup for the measurement of the temporal decay of the memory effect. The first excimer laser initiates the memory effect and the second generates the acoustic pulse. 